\title{
Special Issue: Underwater Communications and Sensors Technologies
}

\author{
Pablo Otero ${ }^{1} \cdot$ Bhawani Shankar Chowdhry ${ }^{2} \cdot$ Huacheng Zeng ${ }^{3} \cdot$ Muhammad Aamir $^{4}$ \\ Published online: 7 January 2021 \\ (c) The Author(s), under exclusive licence to Springer Science+Business Media, LLC part of Springer Nature 2021
}

This special issue is mainly composed of the extended versions of selected papers presented at the third "Global Conference on Wireless and Optical Communications" (GCWOC'18), October 1-3, 2018, Malaga, Spain. GCWOC'18 was jointly organized by the University of Malaga (UMA) Spain, Mehran University of Engineering and Technology (MUET), Jamshoro, Pakistan, and Sir Syed University of Engineering and Technology (SSUET) Karachi. GC-WOC aims to facilitate a creative environment for the promotion of collaboration and knowledge transfer.

Moreover, the issue also includes selected papers following the call for submissions of original research papers, both theoretical and practice-oriented, from academia, industrial professionals, and students from relevant engineering and technology fields with a focus on Underwater Communications and Sensors Technologies.

The first paper titled as "Optical Versus Electrical: Performance Evaluation of Network On-Chip Topologies for UWASN Manycore Processors". The authors assessed the performance of electrical and optical Network On-Chip (NoC) mesh and torus topologies with 64 and 144 nodes. The impact of different packet and network sizes was explored on average latency and throughput of NoCs. The experimental outcomes revealed that ONoC has advantages of improved average latency and improved throughput for large packet size. The authors also proposed a hybrid optical-electrical NoC topology based on multi write single read serpentine optical bus architecture aiming to minimize the communication latency and energy consumption.

In the Second paper, the authors suggested the enhanced model of J48 algorithm based on traditional J48 decision tree method for measurement accuracy and performance. It is entitled as "addresses the indoor localization has become more popular for wireless devices". The proposed approach is viable for both air and underwater communication based on the visible light spectrum. Accuracy, distance error, and processing time were observed by experiment. Considering, accuracy the proposed method yielded $98.0 \%$ with error distance as compared to other classifiers. With regard to training time

Bhawani Shankar Chowdhry

c.bhawani@ieee.org

1 University of Malaga, Malaga, Spain

2 Mehran University of Engineering and Technology, Jamshoro, Pakistan

3 Michigan State University, East Lansing, USA

4 Sir Syed University of Engineering and Technology, Karachi, Pakistan 
this method performed average due to its complexity. It was well depicted that Visible Light Communication (VLC) network can perform with high accuracy for localization based applications.

Third paper titled as "A Traffic Delay and Bandwidth Based Multipath Scheduling Approach for Optimal Routing in Underwater Optical Network". In this paper, a traffic delay and bandwidth based multipath scheduling approach for optimal routing in the optical network was proposed. This aim is to route the traffic effectively using traffic delay computation and selecting dynamic multipath. In most cases, the pre-configured route for a source to a destination gets overloaded if the incoming traffic is increased beyond it bandwidth limit which cause the link blockage. It was predicted for each node that the probability of blockage based on the traffic delay and identifying the most efficient dynamic multipath route based the computed delay value; which enabled the optimal utilization of bandwidth and minimized the bandwidth blockage rate to improvise the throughput. This saving of the Bandwidth gives better results in Under water communication.

The fourth paper entitled "A Broadband High Gain Tapered Slot Antenna for Underwater Communication in Microwave Band". In this research, the authors proposed a broadband high gain Tapered slot antenna array for under water communication. The procedure to design the unit element antenna is followed by applying a linear tapered array-slot structure to the conventional Vivaldi antenna; hence the bandwidth, gain and radiation efficiency of the antenna are improved.

In fifth paper the authors presented an automated system for identification and classification of fish species. It can help the marine biologists to have greater understanding of the fish species and their habitats. The proposed model based on deep convolutional neural networks. It is entitled as "Automatic Fish Species Classification Using Deep Convolutional Neural Networks". It uses a reduced version of AlexNet model comprises of four convolutional layers and two fully connected layers. A comparison presented against the other deep learning models such as AlexNet and VGGNet by considering number of relevant parameters.

The sixth paper proposes Classification of DoS Attacks in Smart Underwater Wireless Sensor Network. This research paper presented a customized dataset for smart underwater wireless sensor network that can be categorized into four types of DoS attacks (Blackhole, Gray hole, Flooding and Scheduling attacks). It is entitled as "Classification of DoS Attacks in Smart Underwater Wireless Sensor Network". For the experimental purpose, a low Energy Aware Cluster Hierarchy protocol utilized in this research work. Using NS2 network simulator model, a scheme had been defined to collect network traffic and create the dataset.

The seventh paper entitled "Vehicle Pollution Monitoring, Control and Challan System Using MQ2 Sensor Based on Internet of Things". The proposed system can help in maintaining the level of gases within the predefined limits. The results show that the system fulfils the requirement of a sensor based embedded system that can screen and control the air contamination with generation of challan from anyplace in the world using IOT. The system can be easily deployed in any vehicle that is contributing in air pollution.

The title of eighth paper is "Wireless Communication as a Reshaping Tool for Internet of Things (IoT) and Internet of Underwater Things (IoUT) Business in Pakistan: A Technical and Financial Review". In this paper, a brief survey on the potential market of ICT in Pakistan and its influence on the economic growth is presented. Moreover, the penetration of Consumer Electronics (CE) business in the local market has also been discussed. The discussion shows that the scope of CE business in Pakistan will be ascended significantly by employing $5 \mathrm{G}$ IoT/IoUT on the CE product. 
The ninth paper entitled "Controlling and Stabilizing the Position of Remotely Operated Underwater Vehicle Equipped with a Gripper". This research work presented controlling and stabilizing the position of ROUV equipped with a gripper. The proportional, integral and derivative (PID) controller is responsible to attain the desired altitude and nonlinear observer model with PID controller used for the perfect tuning of Euler angles with respect to the desired angles related to the desired position. During the fight, variation arises in terms of hydrodynamic disturbances and due to the movements of (2 DOF) gripper. The applied control structure is capable to control the rapid variation and to minimize the error margin.

The tenth paper presented the application of sigma-delta modulation (SDM) in the design of adaptive channel equalizer using the Wiener filter for wireless and underwater acoustic communication (UWA). It is entitled as "Sigma-Delta Modulation Based Adaptive Channel Equalizer Based on Wiener-Hopf Equations". The simulation of the proposed design was done in MATLAB at first and various performance parameters were compared and observed. While in second phase, the same design was implemented in FPGA and the area performance analysis was carried out. The designs seen before in the same domain had some convergence and noise issues. Those were mitigated in this work. Hence this may have concluded that, the use of SDM based Wiener filter implemented on ASICS or FPGAs opens the door of adaptive signal processing in short word length domain.

The eleventh paper titled "Polarization Agile Antenna for Underwater Communication Using Integrated Power Divider and Phase Shifter". The research article presented a design of polarization agile antenna that can be helpful for underwater communication, using the integrated structure of power divider and phase shifter to feed a square patch antenna. In contrast to the traditional DFP polarization agility antenna feed networks adopting the separate power divider and phase shifters, a feed structure integrating the power divider and the phase shifter is employed in this paper with improved performance. This system has reduced the number of switches, simplified the control circuit, and reduced the insertion loss which is suitable candidate for underwater communication.

The title of Twelfth paper is "IoT-Enabled Smart Socket". This paper presented the state-of-the-art technologies that have been proposed in the literature related to IoT-enabled solutions for energy conservation. This paper also highlighted the details of infrastructure and communication models used in the literature for implementing such IoT-enabled applications. Based on the existing literature, this paper identified areas that need more attention and can be served as open issues or gaps for future research. In the end, this paper presented IoT-enabled smart socket, which can be useful to serve the needs of consumers from developing countries like Pakistan.

The 13th paper proposed a testbed which can evaluate the Under Water (UW) communication system in a controlled aquatic environment and simulates the UW channel and sound propagation models. It is entitled as "Design of a Web Based Underwater Acoustic Communication Testbed and Simulation Platform". The constraint of physical access to the testbed facility resolved by using a web-based monitoring and controlling graphical user interface.

The title of 14th paper is "Phase Sensing Technology Based Optical Signal Regeneration for $40 \mathrm{~Gb} / \mathrm{s}$ Optical System". In this paper, phase sensing based optical signal regeneration has been designed and developed for the noisy long haul optical system. The designed phase sensing based optical signal regeneration is implemented for $150 \mathrm{~km}$ and $200 \mathrm{~km}$ noisy SMF fiber link. HNLF fiber is configured for a different length of $0.5 \mathrm{~km}$ and $1 \mathrm{~km}$. After the analysis, it has been defined that the minimum BER with maximum Q-factor have been achieved for both $150 \mathrm{~km}$ and $200 \mathrm{~km}$ using the 
HNLF of $1 \mathrm{~km}$. The main contribution of the work is that using the designed system the optical to electric conversion for regeneration is not used.

The 15th Paper proposed a stream based authentication mechanism that specifically addressed the security concerns of multi-homing sub-aqueous big data networks. It also presented a key authorization infrastructure that performs a proper handing taking among multiple off-ground Data nodes before an inter-network data block exchange. The title of paper is "Stream-Based Authentication Strategy Using IoT Sensor Data in Multi-homing Sub-Aqueous Big Data Network". The simulation results depicted that the proposed approach increases multi-homing network compatibility and reliability while processing a data block in the sub-aqueous distributed computing environment.

The title of 16th paper is "Critical Analysis of Localization and Time Synchronization Algorithms in Underwater Wireless Sensor Networks: Issues and Challenges". This study presented a comprehensive survey about the algorithm that have been designed for the localization and time synchronization in underwater sensors networks. The algorithms are classified in categories and analysis carried out on the basis of challenges faced by localization and time synchronization in UWSNs such as type of sensors, ranging methodology, communication mode, time interval, coverage area, energy consumption and computational complexity.

The 17th paper entitled "Wavelet Techniques for Medical Images Performance Analysis and Observations with EZW and Underwater Image Processing". In this paper, the EZW algorithm has been discussed with Haar wavelet and Bior4.4 techniques on skeleton images with different type of image quality parameters. The analysis shows that the compression ratio (CR) of EZW with Bior4.4 on skeleton image is $44.19 \%$, MSE is 527.73 and PSNR is 45.45. While, the CR of EZW with Haar on the same image is $40.31 \%$, MSE is 699 and PSNR is $35.34 \mathrm{db}$. Further EZW can help precise capacity of amount, exterior area and other morph metric capacity of organic stuff, lacking removing them commencing the sea or in the marine environment.

The title of 18th paper is "Design and Analysis of Variation in Chlorophyll and Depth for Open Ocean Underwater Optical Communication". In this paper, an attempt has been made to study the attenuation coefficient in underwater optical wireless communication through published work. The attenuation coefficient detects at which length light source is vague. The aim of the work is to investigate the change in attenuation at different depth level in underwater application.

In the 19th paper, design of remotely operated under water vehicle is proposed with an aim to control the position and stabilized dynamic behavior of the vehicle by using dual control scheme. The design structure of ROUV consists of six thrusters that help the underwater vehicle to achieve 6-DOF easily. The dual controller is a combination of model adaptive and PID controller. The paper is entitled as "Stabilizing the dynamic behavior and Position control of a Remotely Operated Underwater Vehicle".

The 20th paper presented three efficient meta-heuristic evolutionary algorithms ant colony optimization, artificial bees colony and firefly algorithm, inspired by swarm intelligence are being compared with an objective to achieve the shortest path for the mobile node in traversing the complete sensing network. The title of the paper is "Comparative Analysis of Bio-Inspired Algorithms for Underwater Wireless Sensor Networks".

The 21st paper entitled "State-of-the-Art Underwater Acoustic Communication Modems: Classifications, Analyses and Design Challenges. The main contribution of the article is a comparative analyses of commercial and research modems based on their characteristics and design constraints, in order to describe the current trends and more 
promising techniques. This paper is focused on the state-of the-art underwater acoustic modems designed, developed and implemented in the last few years.

The title of 22nd paper is "Wireless Networks for Voltage Stability Analysis and Anti-islanding Protection of Smart Grid System". This research proposed a framework to analyze the voltage stability and anti-islanding of smart grid system after integration of Distributed Generations (DGs) using modified Thevenien equivalent model and improved Zigbee network respectively. The proposed framework is highly efficient as compared to traditional approaches such as contingency analysis, $\mathrm{P}$ v/s Q approach, vector relays etc.

In the 23rd paper, the authors have reviewed the existing literature on WBAN Reliability enhancements' techniques followed by a review of QoS aware enhancements for AODV. The proposed protocol is designed and simulated for Intra-WBAN communication and is efficient in terms of end-to-end reliability.

The paper is entitled as "QoS Enhancement of AODV Routing for MBANs".

The 24th paper entitled "Fine-Grained Data Processing Framework for Heterogeneous IoT Devices in Sub-aquatic Edge Computing Environment". This paper proposed a finegrained processing framework that normalizes tuple non-classification through enhanced false-positive function and assembles IoT sensory tuples with the in-memory capacity to rectify compression failovers. This solution leads to a tremendous decrease in bundle block placement and end-of-file replica latencies. The simulation results depict the effectiveness of fine-grained processing framework through easing the edge nodes in the sub-aquatic distributed environment.

The title of 25th paper is "Flexible OFDM Transceiver for Underwater Acoustic Channel: Modeling, Implementation and Parameter Tuning". This work proposed a Matlab model of an OFDM transceiver along with UWA channel characterization based on Rician shadowed fading model as it perfectly characterizes the way in which a shallow UWA channel behaves. Eventually, the proposed design allows implementation of various OFDM modulation methods and to perform Monte Carlo simulations for bit error rate comparisons together with the ability to tune multiple UWA channel parameters.

The 26th paper entitled "Cluster Estimation in Terrestrial and Underwater Sensor Networks". In this work, the authors have proposed an analytical estimate which considers sensor node's transmission range and sensing field dimensions for finding the correct number of the clusters in a sensing field. With this approach a better network coverage and connectivity during the exchange of data can be achieved, which in turn increases the network performance.

In the 27th paper, a new smart sensor-based detection technique for the underwater communication signal has been proposed. With the use of the proposed sensing scheme, the detection performance can be improved and it also overcomes sensing failure problem. Simulation results confirm that in the field of detection probability, the proposed scheme beats the existing methods. The title of paper is "Smart Sensor for the underwater communication signal".

The 28th paper entitled "Nodes Deployment Algorithm Based on Data Fusion and Evidence Theory in Wireless Sensor Networks". In this paper, a dynamic network coverage algorithm based on evidence theory is proposed in the aspect of wireless sensor network coverage. The simulation results show that the algorithm can achieve complete coverage of the monitoring area and effectively reduce the movement of nodes.

The title of 29th paper is "Temporal Masking with Luma Adjusted Interframe Coding for Underwater Exploration using Acoustic Channel". In this work, the authors have proposed an algorithm of Temporal Masking with Luma Adjustment to reduce the video information for efficient transmission and reduce the need of over compression. Proposed 
algorithm is applied on the Moving Picture Expert Group video standard. A 2D-Least Mean Squared Adaptive Filter is embedded at the receiver end in order to reduce the effect of aquatic noises and further compensate the overall quality.

We would like to thank the Editor-in-chief (Professor Ramjee Prasad), Mr. Meertinus Faber (Project Coordinator of Production at Springer Nature), and Mr. Joseoh Ian Reyes (Springer's Journal's Editorial Office Assistant) for their support and help in realizing this special issue. Special thanks to all the authors for promptly revising their papers to meet the requirements of reviewers. Thanks to all valued reviewers for contributing with their expert comments to improve selected papers. Hope you will have the benefit by reading this special issue.

Publisher's Note Springer Nature remains neutral with regard to jurisdictional claims in published maps and institutional affiliations.

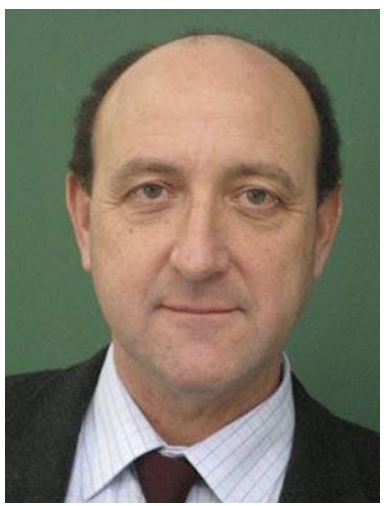

Dr. Pablo Otero received the Ingeniero de Telecomunicacion degree from the Polytechnic University of Madrid (UPM), Madrid, Spain, in 1983, and the Ph.D. degree in electrical engineering from the Swiss Federal Institute of Technology (EPFL), Lausanne, Switzerland, in 1998. From 1983 to 1993, he was with the Spanish companies Standard Electrica, E.N. Bazan, and Telefonica, where he was involved in communication and radar systems engineering. In 1993, he joined the University of Seville, Seville, Spain, where he lectured radio communications and radio wave propagation for two years. In 1996, he joined the Laboratory of Electromagnetism and Acoustics, EPFL, where he was a Research Associate, granted by the Spanish Government. In 1998, he joined the University of Malaga, Malaga, Spain, where he is currently an Associate Professor. His research interests include electromagnetic and antenna engineering and underwater acoustic communications.

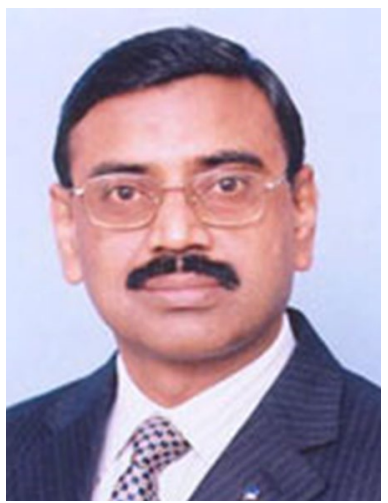

Professor Dr. Bhawani Shankar Chowdhry is the Distinguished National Professor and former Dean Faculty of Electrical Electronics and Computer Engineering, Mehran University of Engineering and Technology (MUET), Jamshoro, Pakistan. He has the honour of being one of the editor of several books "Wireless Networks, Information Processing and Systems", CCIS 20, "Emerging Trends and Applications in Information Communication Technologies", CCIS 281, "Wireless Sensor Networks for Developing Countries", CCIS 366, "Communication Technologies, Information Security and Sustainable Development", CCIS 414, published by Springer Verlag, Germany. He has also been serving as a Guest Editor for "Wireless Personal Communications" which is Springer International Journal. He has produced more than dozen PhDs and supervised more than 50 MPhil/Masters Thesis in the area of ICT. His list of research publication crosses to over 60 in national and international journals, IEEE and ACM proceedings. Also, he has Chaired Technical Sessions in USA, UK, China, UAE, Italy, Sweden, Finland, Switzerland, Pakistan, Denmark, and Belgium. He is member of various professional bodies including: Chairman IEEE Communication Society (COMSOC), Karachi Chapter, Region10 Asia/Pacific, Fellow IEP, Fellow IEEEP, Senior Member, IEEE Inc. (USA), SM ACM Inc. (USA). He is lead person at MUET of several EU funded Erasmus Mundus Program including "Mobility for Life", "StrongTies", "INTACT", and "LEADERS". He has organized and coorganized several International Conferences including "IMTIC08", "IMTIC12", "IMTIC13", "IMTIC15", 
"IMTIC18" "WSN4DC13", "IEEE SCONEST", "IEEE PSGWC13”, "GCWOC15", "GCWOC16", "GCWOC17", "GCWOC18” and track chair in "Global Wireless Summit (GWS 2014).
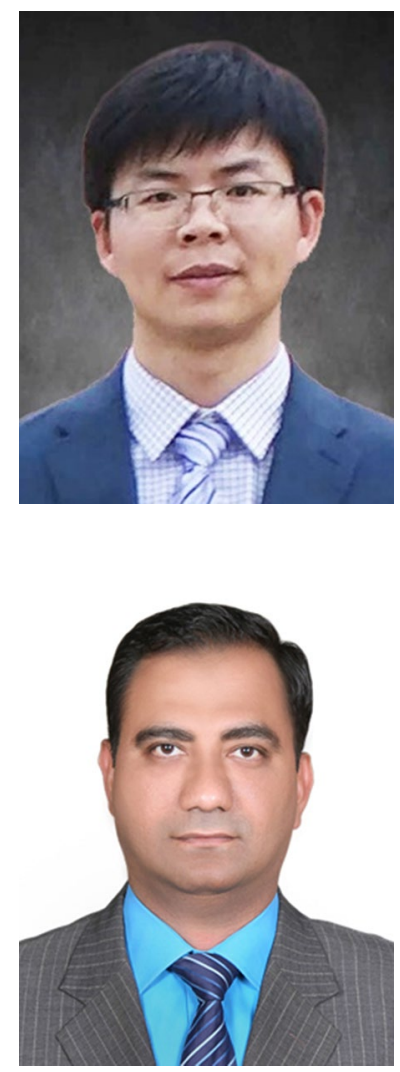

Huacheng Zeng $\mathrm{He}$ is an Assistant Professor in the Department of Computer Science and Engineering at Michigan State University (MSU). Prior to joining MSU he was working as an assistant professor in the Department of Electrical and Computer Engineering at the University of Louisville (UofL). Before joining UofL, he worked as a senior system engineer at Marvell Semiconductor, Santa Clara, CA, from March 2015 to August 2016. He received his Ph.D. degree in Computer Engineering from Virginia Tech in 2015, and his B.S. and M.S. degrees from Beijing University of Posts and Telecommunications, Beijing, China, in 2007 and 2010, respectively. His research interests lie in advanced wireless technologies and innovative wireless applications. Dr. Zeng is a frequent reviewer for IEEE journals such as Transactions on Networking, Selected Areas in Communications, Transactions on Wireless Communications, and others.

Prof. Dr. Muhammad Aamir received MS degree in Electronic Engineering (with specialization in Telecommunication) in 2002 and BS in Electronics Engineering in 1998. He accomplished his PhD in Electronic Engineering from Mehran University of Engineering and Technology. During his Ph.D. studies, he accomplished his research work at the University of Malaga under Erasmus Mundus Scholarship. He has authored and co-authored more than 50 research papers and book chapters published in various journals, books and conferences of international repute. He is a life member of Pakistan Engineering Council and professional member of IEEE for last 12 Years. He was also awarded with a grant by the Ministry of Education Spain to teach at the University of Malaga, which he availed in May 2012. He is also Member of NCRC in the Higher Education Commission (HEC) for revision of Electronic Engineering Curriculum and Telecommunication Engineering Curriculum at the National Level. He is also HEC approved supervisor for $\mathrm{PhD}$ candidates. He is currently serving as Associate Dean Faculty of Electrical and Computer Engineering. 